albicans had resistance to a specific antifungal: 2 to fluconazole, 1 to flucytosine, 4 to itraconazole and 1 to voriconazole. No samples of Candida parapsilosis had resistance to any of the antifungals tested, however 16 had intermediate resistance to caspofungin.

Conclusions This 15 year study of candidaemia in a tertiary paediatric hospital shows that almost $80 \%$ of the cases were due to Candida albicans or Candida parapsilosis. Resistant Candida species and overall resistance to antifungal medication was uncommon and did not show any increasing trend over time.

\section{G54 IMPACT OF PNEUMOCOCCAL CONJUGATE VACCINES ON PNEUMOCOCCAL MENINGITIS IN ENGLAND AND WALES, $2000-2016$}

\begin{abstract}
${ }^{1,2} \mathrm{G}$ Oligbu, ${ }^{3} \mathrm{~A}$ Djennad, ${ }^{1} \mathrm{~S}$ Collins, ${ }^{4} \mathrm{CL}$ Sheppard, ${ }^{4} \mathrm{NK}$ Fry, ${ }^{5} \mathrm{R}$ Borrow, ${ }^{3} \mathrm{NJ}$ Andrew, ${ }^{1,2} \mathrm{~S}$ Ladhani. ${ }^{1}$ Paediatric Infectious Diseases Research Group, Institute for Infection and Immunity, St George's, University of London, UK; ${ }^{2}$ Immunisation, Hepatitis and Blood Safety Department (IHBSD), Public Health England, London, UK; ${ }^{3}$ Statistics, Modelling, and Economics Department, Public Health England, London, UK; ${ }^{4}$ Respiratory and Vaccine Preventable Bacterial Reference Unit (RVPBRU), Public Health England, London, UK; ${ }^{5}$ Vaccine Evaluation Unit, Public Health England, Manchester Medical Microbiology Partnership, Manchester Royal Infirmary, Manchester, UK
\end{abstract}

\subsection{6/archdischild-2018-rcpch.52}

Introduction The introduction of pneumococcal conjugate vaccines (PCV) was associated with reduction in incidence of invasive pneumococcal disease (IPD) especially IPD caused by the vaccine serotypes. Its impact on meningitis in the United Kingdom has not been assessed.

Methods Public Health England conducts enhanced surveillance for IPD and provides a national reference service for serotyping pneumococcal isolates in England and Wales. Data were extracted for isolates from confirmed IPD cases between 1 st July 2000 and 30th June 2016, covering the 2000/01 to 2015/16 epidemiological years. Incidence rate ratio (IRR) and case fatality rate (CFR) were calculated. Multivariable logistic regression was used to calculate the odds of meningitis and assessed its association with death.

Results There were 80313 laboratory-confirmed IPD cases over the 16 year surveillance period, including 4160 cases (4.9\%) with meningitis. Of the 4108 with reported age, 1611 $(39.2 \%)$ cases were reported in children aged $<5 \mathrm{y}, 1729$ (42.1\%) in 5-64 year-olds and $768(18.7 \%)$ cases in 65+year olds. This compares with 8324 (10.5\%), 32297 (40.6\%) and $38999(49.0 \%)$ of 79620 non-meningitis cases during the same period, respectively $(\mathrm{p}<0.001)$.

PCV7 introduction in September 2006 had no impact on the overall incidence of pneumococcal meningitis $(0.55 / 100,00$ during 2000/01-2005/06 vs $0.56 / 100,000$ during 2008/092009/10) because of serotype replacement disease. PCV7 replacement with PCV13 in April 2010, however, led to a 48\% (95\% CI: 38\%-62\%) reduction in pneumococcal meningitis incidence by 2015/16, whilst meningitis cases due to non-PCV13 serotypes remained static.

The overall CFR was $17.5 \%(631 / 3,611$, increasing from $10.7 \%(150 / 1408)$ in $<5$ y to $17.3 \%(262 / 1517)$ in $5-64$ y and $31.9 \%(219 / 686)$ in $65+$ year olds. This compared with $3.6 \%(254 / 716), 10.8 \%(3,235 / 30,090)$ and $30.6 \%(11,292 /$ $36,907)$ for non-meningitis for the same age groups, respectively. CFR for meningitis due to PCV7 serotypes (130/ 916, 14.2\%) compared to PCV13 (143/793, 18.0\%) or nonPCV13 serotypes (290/1,534, 18.9\%). Among meningitis cases, serotype 8 was associated with increased odds of death (aOR, 2.91; 95\% CI: 1.79 to $4.71 ; \mathrm{p}<0.0001)$

Conclusions The impact of PCV on pneumococcal meningitis has been less prominent than for other IPD presentations and case fatality remains high; a different strategy is, therefore, required to reduce the burden and outcomes of pneumococcal meningitis.

\section{G55 EVALUATING THE RISK OF LACERATION WHEN USING AN ADRENALINE AUTO-INJECTOR TO TREAT ANAPHYLAXIS VIA THE TWO STANDARD METHODS OF ADMINISTRATION}

${ }^{1} \mathrm{~L}$ Pike, ${ }^{2} \mathrm{D}$ Tuthill. 'School of Medicine, Cardiff University, Cardiff, UK; ${ }^{2}$ Department of Paediatrics, Noah's Ark Children's Hospital of Wales, Cardiff, UK

\subsection{6/archdischild-2018-rcpch.53}

Aims Anaphylaxis is a severe and potentially fatal, systemic allergic reaction. Adrenaline is the treatment of choice, usually given via an adrenaline auto-injector (AAI). Concerns over lacerations to young children moving their leg whilst being given an AAI by the recommended 'swing and jab' ( $S$ and J) method led to this service evaluation of the two methods of administering AAIs; $\mathrm{S}$ and $\mathrm{J}$ and 'push and press' ( $\mathrm{P}$ and $\mathrm{P}$ ).

Methods A training EpiPen ${ }^{\circledast}$ and paint was used to evaluate the methods. Paint was placed in the centre of the training pen, any excess removed from the 'needle' indentation and left flush with the bottom of the pen. Children aged 5-11 had both methods 'administered' to their outer thigh on bare skin. They were asked to move their leg when the pen fired in order to simulate real injections. The method used first was alternated between successive participants. Age, movement and length of paint mark $( \pm 0.1 \mathrm{~mm})$ were recorded. The mean measurement of paint marks made by no movement was calculated and subtracted from all measurements leaving the distance the AAI moved. Analysis was conducted using IBM $^{\circledast}$ SPSS $^{\circledast}$ Statistics version 23.

Results 135 children (mean age 8 years) were asked to take part; measurements were taken from 100 (74\%). 50 children (50\% of participants) moved for one or both methods; 32 (32\%) moved for both methods. 18 (18\%) moved for either S and $\mathrm{J}$ (12 children), or P and P (6 children). The number of children who moved for each method was significantly different (chi-squared: $\mathrm{p}=0.033$ ). $\mathrm{S}$ and $\mathrm{J}$ had a mean movement of $8.3 \mathrm{~mm}$ (95\% CI: 3.4 to 13.3$) ; \mathrm{P}$ and $\mathrm{P}$ had a mean of $3.5 \mathrm{~mm}$ (95\% CI: 0.4 to 6.6). The mean difference between methods for those that moved was $4.8 \mathrm{~mm}$ (95\% CI: 1.9 to 7.7) and paired samples T-test showed $\mathrm{p}=0.001$.

Conclusion This evaluation showed a statistically significant difference in the length of marks made by $S$ and $J$ and $P$ and P. Previous cases showed that there is a risk of laceration when administering EpiPen ${ }^{\circledR}$ to young children using the recommended 'swing and jab' method and therefore this evaluation suggests it may be advisable to change to teaching 'push and press' to carers who administer adrenaline auto-injectors to young children. 